\title{
Effects of ischemic preconditioning on indirect markers of exercise-induced muscle damage: protocol for a randomized placebo-controlled trial.
}

\author{
Mikhail Santos Cerqueira ${ }^{1 *}$, Ingrid Martins de França1, Mauro Bezerra Montello', Daniel Kovacs', \\ Wouber Hérickson de Brito Vieira'
}

\begin{abstract}
Background: Ischemic preconditioning (IPC) has been used to improve exercise performance, but its role in protecting against exercise-induced muscle damage (EIMD) is still unclear. Objective: To investigate the effects of IPC on the indirect markers of EIMD when compared to placebo. Methods: 30 healthy young men, with no recent experience in lower limb strength training, will be recruited. Subjects will be allocated randomly into two groups: IPC or placebo. The IPC group will undergo $4 \times 5$ min of occlusion (with individualized total occlusion pressure), interspersed with 5 min of reperfusion. The placebo group will be submitted to the same protocol, but with minimum pressure $(10 \mathrm{mmHg})$ being applied during the occlusion period. After the interventions, volunteers will be submitted to muscle damage induced by isokinetic exercise (10 sets of 12 maximum eccentric repetitions) in the non-dominant femoral quadriceps. The primary outcome will be isometric peak torque, measured both before and up to 72 hours after exercise. Secondary outcomes include rate of torque development, muscle soreness, knee range of motion, thigh circumference and blood levels of creatine kinase. Discussion: The results of this trial will indicate whether the effects of IPC are superior to placebo in the protection against EIMD.

Keywords: Blood Flow Restriction; Prevention; Eccentric Exercise; Muscle Recovery; Placebo Effect.
\end{abstract}

\section{INTRODUCTION}

Exercise-induced muscle damage (EIMD) is a phenomenon that occurs as a result of novel or unaccustomed exercise. It is characterized by loss of strength and range of motion, delayed-onset muscle soreness (DOMS), swelling and increase in blood concentrations of creatine kinase ${ }^{(1,2)}$. Considering that muscle damage can impair muscle performance and adherence to exercise programs, therapeutic methods such as massages, photobiomodulation, cryotherapy, and compressive sleeves are commonly employed to accelerate post-exercise recovery and maintain training intensity and frequency ${ }^{(3-7)}$. Alternatively, some pre-exercise interventions have been used to minimize muscle damage ${ }^{(8,9)}$. A method which has been recently proposed to protect against EIMD is ischemic preconditioning (IPC) ${ }^{(10)}$, which consists of periods of total vascular occlusion followed by reperfusion, induced through insufflations and deflations of a pressure cuff(11,12). IPC can have both acute (immediately after, lasting up to 4 hours) and late (12 to 72 hours after) repercussions ${ }^{(12,13)}$. The acute effects of IPC have been extensively investigated in studies which evaluated performance in the first few hours after ischemic stimulus. However, few studies have investigated the late effects of IPC on skeletal muscle ${ }^{(11)}$.

Elevated intramuscular acidosis as well as increases in reactive oxygen species and immune activity are common responses to both IPC and damaging exercise ${ }^{(10)}$. It has been proposed that the late effects of IPC may contribute to protect against EIMD through the improvement of metabolic efficiency secondary to increased mitochondrial electron flow, attenuation of ATP depletion, and increased blood flow due to elevated adenosine levels post-ischemia ${ }^{(13-15)}$. In fact, a recent study ${ }^{(16)}$ has found that IPC ( $3 \times 5$ minutes of ischemia interspersed with 5 minutes of reperfusion; occlusion pressure of $200 \mathrm{mmHg}$; 8 -cm-wide cuff) applied immediately prior to an eccentric muscle damage protocol for elbow flexor muscles ( $3 \times 10$ repetitions with load $80 \%$ of $1 \mathrm{RM}$ ) was able to blunt muscle damage responses. However, it should be noted that, due to some methodological issues, the real effectiveness of using IPC to mitigate muscle damage is still unclear. First, since the control group did not undergo any interventions prior to the muscle damage protocol ${ }^{(16)}$, it is difficult to conclude

\footnotetext{
Study registry: This protocol was published on the Registro Brasileiro de Ensaios Clínicos (REBEC) with the registration number RBR- 8brxg7.

* Corresponding author: Mikhail Santos Cerqueira. Address: Department of Physical Therapy, Universidade Federal do Rio Grande do Norte, Av. Senador Salgado Filho,

3000 - Campus Universitário, Lagoa Nova, Natal, Rio Grande do Norte 59072-970, Brazil; Telephone number: +55 (84) 3342-2018. Email: mikalsantosc@hotmail.com

1 Laboratory of Muscle Performance, Department of Physical Therapy, Universidade Federal do Rio Grande do Norte (UFRN), Natal, RN, Brazil.
}

Financial Support: nothing to declare.

Submission date 26 April 2019; Acceptance date 07 November 2019; Publication date 03 December 2019 
whether the IPC effects are superior to placebo. This is a frequent question and some studies have shown that the acute effects of IPC might not be superior to placebo for performance improvement ${ }^{(15,17)}$. Second, few studies on IPC were concerned with individualizing the occlusion level. It has been proposed that a sufficient occlusion pressure to completely block blood flow should be applied to the member undergoing IPC ${ }^{(11)}$, but the use of arbitrary pressures may induce different stimuli between subjects due to individual factors such as limb circumference and systemic blood pressure ${ }^{(18,19)}$. Nevertheless, even if the usage of arbitrary pressure is enough to totally occlude the blood flow in all subjects ${ }^{(20)}$, the occlusion level may exceed by far the minimum amount necessary to fully occlude blood flow in some participants, thus raising the perception of pain or discomfort during IPC.

Third, it is unclear whether IPC is enough to mitigate reductions in torque and rate of torque development (RTD) after EIMD. Although Franz et al. ${ }^{(16)}$ have evaluated muscle contractility through tensiomyography, peak torque is considered an important functional marker of muscle damage ${ }^{(21)}$. In addition, RTD is derived from the analysis of the torque curve recorded during an explosive voluntary contraction and can provide important physiological information about the influence of muscle damage in both neural (analysis of the first $50 \mathrm{~ms}$ ) and muscular (analysis between $100 \mathrm{~ms}$ and $200 \mathrm{~ms}$ ) function $^{(22,23)}$. Finally, considering that the magnitude of the EIMD is lower in the thigh muscles compared to the arm muscles ${ }^{(2)}$, it is relevant to investigate the effects of IPC in the knee extensors, since Franz et al. ${ }^{(16)}$ studied the effects of IPC in upper limbs only.

Thus, our study aims to investigate the effects of IPC when compared to placebo preconditioning on the indirect markers of exercise-induced muscle damage in knee extensors muscles. Accordingly, the study hypothesis is that IPC will be more effective than placebo in protecting against EIMD.

\section{METHODS}

\section{Study design}

This is a prospectively, two-arm randomized placebocontrolled trial with concealed allocation, blinded measurers and volunteers and intention-to-treat analysis. The protocol was developed according to the SPIRIT (Standard Protocol Items: Recommendations for International Trials) 2013 checklist guidelines $^{(24)}$.

\section{Eligibility Criteria}

Our study will include healthy men aged between 18 and 35 years and classified as irregularly active or active in International Physical Activity Questionnaires (IPAQ). Subjects will be considered eligible if they:
- have not participated in lower-limb strengthening programs in the past 3 months;

- have not had previous experiences with vascular occlusion;

- have a body mass index between 18.5 and $30 \mathrm{~kg} / \mathrm{m}^{2}$;

- have not used vasoactive medications or nutritional supplements regularly;

- have not had lower limb musculoskeletal injuries in the past 3 months;

- do not have diabetes mellitus or uncontrolled blood pressure and are not smokers;

- do not have any inflammatory rheumatologic conditions, severe cardiovascular and/or pulmonary disease that prevents them from performing the evaluation and exercise protocol;

- do not have any psychiatric illnesses and/or malignant tumors and have not contracted Zika or Chikungunya in the last year.

Participants will be excluded if they:

- wish to leave the study;

- present a health problem that disallows continuity in the study;

- use any drugs, electrotherapies, or other therapeutic resources that might interfere in any outcomes;

- perform any unusual or strenuous physical activities during the study period.

Participants will be instructed not to perform any physical exercise or to use any therapeutic form of pain relief or performance improvement during the period of our study. They will also be instructed not to consume any alcohol during the procedure period.

\section{Experimental procedures}

Each participant will visit the lab five times. In the first visit, we will assess personal and anthropometric data (age, height, total body mass and body mass index), physical activity level (by the IPAQ), systemic artery pressure and total occlusion pressure (TOP). In the second visit, which will occur from three to five days after the first one, we will assess systemic arterial pressure, perception of quadriceps pain through the visual analogue scale (VAS), and collect blood samples in order to measure CK levels. We will also assess knee range of motion (ROM), thigh circumference, quadriceps pain threshold, and maximal voluntary isometric contraction (MVIC) of the knee extensor muscles. After the MVIC evaluation, we will apply the pre-conditioning intervention (IPC or placebo), followed by the EIMD protocol. MVIC will be evaluated again immediately after EIMD. In the following sessions (24, 48 and 72 hours after EIMD), volunteers will return for 
the collection of new blood samples as well as evaluation of the perception and threshold of quadriceps pain, thigh circumference, range of motion and MVIC. Evaluations will always be performed at the same time in order to minimize any influences of the circadian cycle. The study design is shown in Figure 1.

\section{Ethical aspects}

This study was approved by the Research Ethics Committee of Universidade Federal do Rio Grande do Norte (CAAE: 94024818.2.0000.5537). All participants must agree and sign an informed consent form in order to be included. The trial was prospectively registered at the Registro Brasileiro de Ensaios Clínicos (RBR-8brxg7).

\section{Randomization and allocation procedures}

Participants will be randomly assigned to the IPC or placebo group. The randomization will be performed through a random numerical sequence generated in the website www. randomization.com. Participant allocation will be concealed in sequentially numbered and sealed opaque envelopes prepared before the study begins. A researcher, who will not be involved in the various stages of the study, will be responsible for the randomization and allocation procedures. The researchers responsible for assessing outcome measures will not have access to the volunteer allocation nor be present at the time of preconditioning. Researchers responsible for determining the TOP and the application of IPC or placebo will not participate in the evaluation of outcome measures or volunteers allocation. The researcher responsible for the evaluation of the outcomes will be masked regarding the applied preconditioning.

\section{Total occlusion pressure determination}

The TOP of the posterior tibial artery will be determined at rest through portable vascular Doppler (DV 6010B Medmega, Franca - SP). All participants will be instructed to avoid strenuous exercise and alcohol intake within the
48 hours prior to the TOP evaluation. Before determining TOP, subjects will be placed in a relaxed dorsal decubitus position in a climatized and silent room (23ㅇ) for ten minutes. Participants will stay in a supine position with arms and legs at rest on the exam table. During TOP determination, the Doppler transducer will be positioned at the mean distance between the medial malleolus of the tibia and the Achilles tendon. The pressure cuff (Aneroid sphygmomanometer Premium, Duque de Caxias-RJ; cuff width $20 \mathrm{~cm}$ ) will be positioned around the sub-inguinal region in the non-dominant upper thigh and inflated based on a prior protocol ${ }^{(18)}$. TOP will be defined as the pressure at the time when arterial pulse is abolished, which will be indicated by the absence of auscultatory signal.

\section{Primary Outcome}

\section{Maximum voluntary isometric contraction}

Initially, participants will be positioned in the isokinetic dynamometer chair (Biodex Multi-Joint System 3, Biodex Biomedical System Inc ${ }^{\circledR}$, New York, USA) following the recommendations of the equipment manufacturer and with the dynamometer rotation axis aligned with the rotation axis of the knee joint (lateral epicondyle of the femur). The MVIC of the knee extensor muscles in the non-dominant limb will be preceded by unilateral warm-up with submaximal isometric contractions (three repetitions lasting six seconds each) in all evaluation days. After warming up, three MVICs will be performed with the knee flexed at $600(0 \circ=$ total knee extension). Participants will be carefully instructed to contract as fast and as strong as possible after the verbal command "go", to maintain the contraction for five seconds and to relax after the verbal "stop" command ${ }^{(25)}$. A strong verbal command will be offered by the researchers and there will be a 30s interval between each MVIC to minimize possible effects of fatigue.

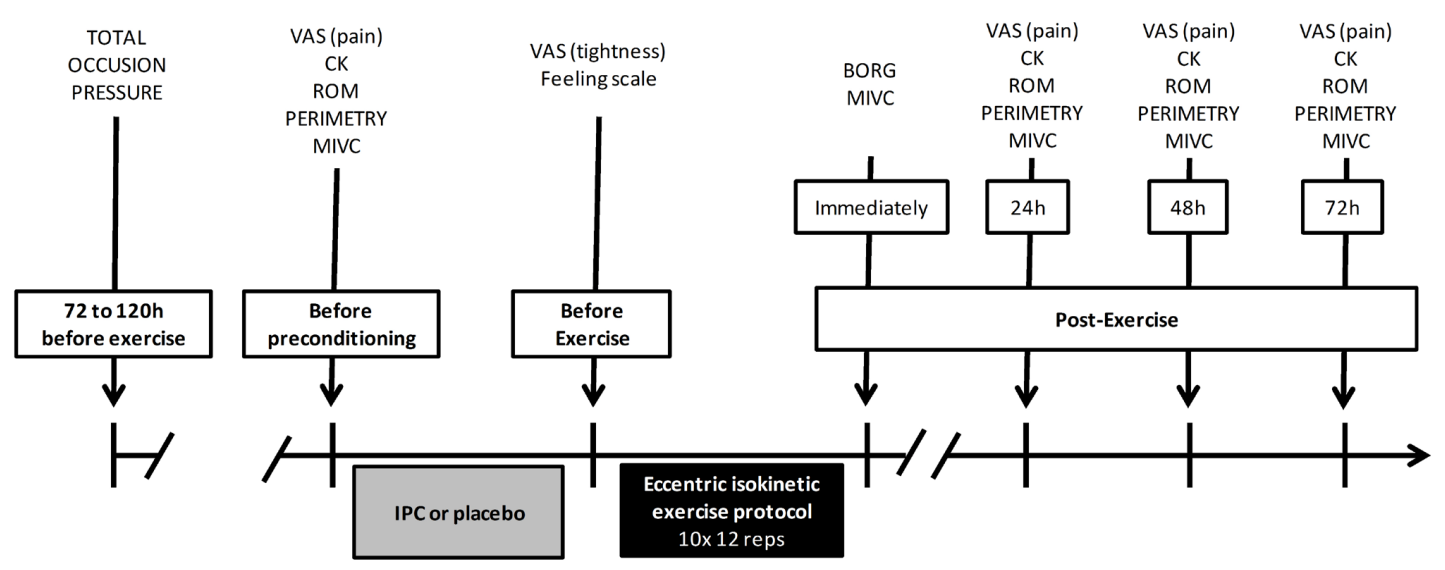

Figure 1. Study Design. Note: VAS: visual analogue scale; CK: creatine kinase; ROM: range of motion; MIVC: maximal isometric voluntary contraction; IPC: ischemic preconditioning. Adapted of Franz et al $^{16)}$. 


\section{Secondary Outcomes}

\section{Rate of torque development}

The rate of torque development (RTD) will be extracted from the isometric torque curve of the evaluated muscles. RTD provides physiological information such as the role of neural and muscular factors in producing strength and neuromuscular fatigue. Analysis of the RFD will be conducted as proposed previously(25).

\section{Muscle soreness}

The visual analogue scale (VAS), consisting of a $100-\mathrm{mm}$ line anchored by a "no pain" descriptor at one end $(0 \mathrm{~mm})$ and a "very, very painful" descriptor at the other $(100 \mathrm{~mm})$, will be used to assess DOMS in the anterior thigh. Subjects will be asked to indicate the perceived pain on the line immediately after the measurement of knee flexion ROM and quadriceps MVIC of the non-dominant limb. The pain level will be determined by the distance between the starting point and the point marked by the volunteer ${ }^{(26)}$. Pressure pain threshold (PPT) will be evaluated through algometry (Wagner Force TenTM FDX50; Wagner Instruments, Greenwich, CT, USA; $1 \mathrm{~cm}^{2}$ pressure tip). The PPT will be evaluated in the non-dominant rectus femoris muscle at points corresponding to $50 \%$ (from proximal to distal) of the distance between the anterior superior iliac spine and the base of the patella. The tip of the algometer will be positioned perpendicular to the evaluation points and pressure will be applied to the skin surface with progressive increases of $1 \mathrm{kgf}$ until the volunteer report that the pressure began to feel painful. An average will be extracted from three measurements for each evaluation point.

\section{Creatine kinase}

A licensed practitioner will collect approximately $10 \mathrm{~mL}$ of venous blood from the antecubital vein. Blood samples will be centrifuged for 10 minutes in order to separate the plasma to be stored at $-80^{\circ} \mathrm{C}$. Serum CK activity will be measured through an enzymatic kinetic assay method (Labtest, Minas Gerais, Brasil).

\section{Range of motion}

Active knee ROM will be measured using a plastic goniometer. The subjects will be placed in ventral decubitus on the examining table and will actively flex their knees as far as possible. Measures will be obtained at peak flexion by measuring the angle between the fibula and the midline of the femur. We will then calculate the average of the three ROM measurements ${ }^{(2)}$.

\section{Thigh Circumference}

Thigh circumference will be evaluated using a measuring tape. Measurements will be performed on the non-dominant limb from the halfway point of the distance measured from the anterior superior iliac spine to the base of the patella. This assessment will be performed in an orthostatic position, with the thigh muscles relaxed ${ }^{(27)}$. Three measurements will be taken per day and the average of each day will be used in the analysis.

\section{Ischemic preconditioning}

After the pre-exercise MVIC, IPC will be applied around the sub-inguinal region of the non-dominant limb with the subjects relaxed and comfortably positioned in supine decubitus on the exam table. IPC will have a total duration of 40 minutes (figure 2), with four cycles of five minutes of total occlusion (TOP determined individually) interspersed with four cycles of five minutes of reperfusion $(0 \mathrm{mmHg})^{(28)}$, and will be performed with the same cuff used to determine TOP.

\section{Placebo preconditioning}

Placebo will be performed on the non-dominant thigh with the same cuff, similarly to IPC (figure 2), but instead with four cycles of five minutes of placebo occlusion $(10 \mathrm{mmHg})$ alternated with four cycles of five minutes of reperfusion $(0 \mathrm{mmHg})^{(28,29)}$. In both groups, subjects will be previously informed that the applied occlusion pressure will be enough to improve performance and prevent muscle damage. In addition, the procedures will be performed in an individualized manner,

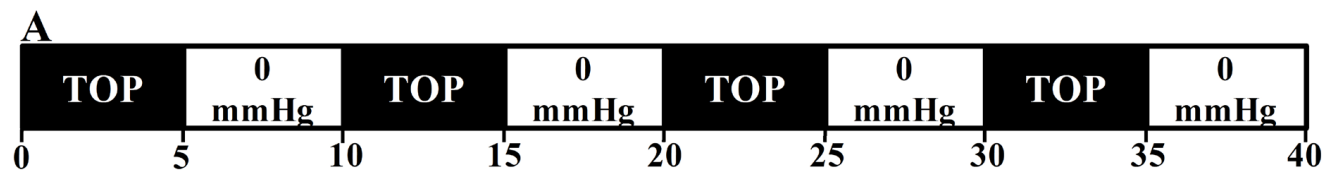

Time (minutes)

B

\begin{tabular}{|c|c|c|c|c|c|c|c|}
\hline $\begin{array}{c}10 \\
\mathrm{mmHg}\end{array}$ & $\begin{array}{c}\mathbf{0} \\
\mathrm{mmHg}\end{array}$ & $\begin{array}{c}10 \\
\mathrm{mmHg}\end{array}$ & $\begin{array}{c}0 \\
\mathrm{mmHg}\end{array}$ & $\begin{array}{c}10 \\
\mathrm{mmHg}\end{array}$ & $\begin{array}{c}0 \\
\mathrm{mmHg}\end{array}$ & $\begin{array}{c}10 \\
\mathrm{mmHg}\end{array}$ & $\begin{array}{c}0 \\
\mathrm{mmHg}\end{array}$ \\
\hline
\end{tabular}

Time (minutes)

Figure 2. Design of ischemic preconditioning (A) and placebo preconditioning (B) protocols. Note: TOP: total occlusion pressure. Adapted of Barbosa et al.28). 
in order to avoid subjects commenting on each other about the compression generated by the cuff.

\section{Damaging Exercise}

Before the EIMD, subjects will perform a warm-up routine (5 minutes of moderate running). After warm-up, the subjects will undergo familiarization (from three to five submaximal isokinetic eccentric actions of the knee extensor muscles) with the EIMD protocol. Three minutes after familiarization, subjects will then perform 120 maximal eccentric actions (10 sets of 12 repetitions, 30 seconds interval between sets) using the non-dominant knee extensor muscles. Before each contraction, the volunteers' knee will be passively positioned at 30 o of flexion ( 0 o=total extension). The subjects will be instructed to perform a brief knee extension to trigger the dynamometer, which will then push the segment up to 90 o of knee flexion (total range of $60 \%$ ) at an angular velocity of $60 \% / \mathrm{sec}^{(30)}$ The subjects will be instructed to resist against the knee flexion movement imposed by the dynamometer as much as possible. Each eccentric action will last for $1 \mathrm{~s}$, followed by $1 \mathrm{~s}$ of passive limb extension. Three minutes after completing the EIMD protocol, the participant's knee will be positioned at 60 을 and a new MIVM will be performed to assess the peak torque after damaging exercise ${ }^{(30)}$.

\section{Perceived discomfort and pleasure/displeasure feeling during interventions}

Perceived discomfort (tightness) will be assessed using the VAS (as described in the evaluation of muscle pain) after IPC or placebo. The pleasure/displeasure feeling regarding each intervention (IPC or placebo) will be assessed using the Feeling scale, a 11-point scale ranging from -5 (very poor) to +5 (very good). The Feeling scale will be applied after assessing perceived discomfort, and the participants will be instructed as follows: "Use the numbers on this scale to indicate how you felt during the intervention. If you felt that the intervention was very good (pleasant or comfortable), then the corresponding answer will be +5 . If the sensation was very bad (unpleasant or uncomfortable), then the corresponding answer will be -5 . If you are feeling in a neutral way (between pleasure and displeasure), then the corresponding answer will be 0 . According to your perception, your answer may also be at an intermediate point between the extremities."

\section{Perceived exertion}

The rating of perceived exertion will be measured using the modified Borg scale (CR10) $)^{(31)}$, which ranges from 0 ("resting state") to 10 ("maximum effort") during EIMD. Subjects will be shown the Borg scale immediately after the EIMD protocol. Participants will be instructed to focus on the level of effort that they exerted to carry out the entire protocol.

\section{Simple size}

A sample size of 30 subjects (15 per group) was calculated based on a clinical trial with a similar experimental design to the present protocol(32). The parameters utilized to sample size calculation included an effect size of 1 , an alpha of 0.05 , power $(1-\beta)$ set at 0.80 , and a difference of at least $10 \%$ in peak torque recovery between the IPC and placebo groups three days after induced muscle damage.

\section{Statistical analysis}

Data normality will be evaluated using the Kolmogorov-Smirnov test. In case of normal distribution, a two-way ANOVA with repeated-measures along with Bonferroni post hoc will be used to assess significant main effects (group, time) and interactions. The mean difference between groups, 95\% confidence interval, and effect size (Cohen's f) will be reported. The Kruskal-Wallis test will be used in case of non-normal data distribution, and the effect size will be calculated using Cohen's $r$. All participants will be included in the analysis following an intention-to-treat approach.

\section{DISCUSSION}

This trial will examine the effects of IPC on the indirect markers of EIMD in the knee extensor muscles. Although a previous study ${ }^{(16)}$ did find positive effects, the absence of a placebo group makes it difficult to establish the real effectiveness of IPC in protecting against muscle damage. In addition, it is unclear whether the IPC can attenuate torque reduction, which is considered an important functional marker of muscle damage. Another concern in our study is verifying perceived discomfort (tightness) and pleasure/displeasure during interventions. These outcomes will add new information that may help to improve adherence to IPC protocols. Thus, from a clinical perspective our results will indicate whether IPC is a useful intervention to protect against muscle damage in physical training and rehabilitation environments. Our study can be considered of high methodological quality because it is randomized and prospectively registered, masks the evaluators and patients, conceals allocation, and uses an intention-to-treat approach. We also calculated sample size in order to provide adequate statistical power to identify possible differences in the main outcomes. Finally, individualizing TOP allows all subjects to receive a similar stimulus while also minimizing possible discomfort due to the unnecessary application of very high occlusion pressures. This protocol is not free of limitations. It is important to consider that the muscular damage promoted in the isokinetic dynamometer may not represent functional situations. Another limitation of the study is the impossibility of masking the researcher who applies IPC. In conclusion, the results of this trial may indicate whether the effects of IPC are superior to placebo in the protection against EIMD. 


\section{PROGRESS OF THE CLINICAL TRIAL}

Data is currently being collected.

\section{Acknowledgements:}

MSC would like to thank Coordenação de Aperfeiçoamento de Pessoal de Nível Superior-Brasil (CAPES), finance code 001, for the scholarship concession.

\section{AUTHOR'S CONTRIBUTIONS:}

MSC: provided the idea for the research and created the hypothesis; MSC, DK and IMF: contributed to the bibliographic research; WHBV, MSC and IMF: critical review of the manuscript. All authors read and approved the final manuscript.

\section{CONFLICT OF INTEREST:}

nothing to declare.

\section{REFERENCES}

1. McHugh MP. Recent advances in the understanding of the repeated bout effect: The protective effect against muscle damage from a single bout of eccentric exercise. Scand. J Med. Sci. Sports. 2003;13:88-97.

2. Chen TC, Lin KY, Chen HL, Lin MJ, Nosaka K. Comparison in eccentric exercise-induced muscle damage among four limb muscles. Eur. J Appl. Physiol. 2011;111:211-223.

3. Bishop PA, Jones E, Woods AK. Recovery from training: a brief review. J Strength Cond. Res. 2008; 22:1015-1024.

4. Cerqueira MS, Santos Borges L, dos Santos Rocha JA, et al. Twelve hours of a compression sleeve is not enough to improve the muscle recovery of an exercise-damaged upper arm. Apunts Med. de l'Esport. 2015;185:23-28.

5. Nampo FK, Cavalheri V, Dos Santos Soares F, de Paula Ramos S, Camargo EA. Low-level phototherapy to improve exercise capacity and muscle performance: a systematic review and meta-analysis. Lasers Med. Sci. 2016;9:1957-1970.

6. Howatson $\mathrm{G}$, Van Someren KA. The prevention and treatment of exerciseinduced muscle damage. Sports Med. 2008;38:483-503.

7. Borges LS, Cerqueira MS, Dos Santos Rocha JA, et al. Light-emitting diode phototherapy improves muscle recovery after a damaging exercise. Lasers Med. Sci. 2014;29:1139-1144.

8. Chen TCC, Chen HL, Pearce AJ, Nosaka K. Attenuation of eccentric exerciseinduced muscle damage by preconditioning exercises. Med. Sci. Sports Exerc. 2012;44:2090-8.

9. Toma RL, Oliveira MX, Renno ACM, Laakso EL. Photobiomodulation (PBM) therapy at $904 \mathrm{~nm}$ mitigates effects of exercise-induced skeletal muscle fatigue in young women. Lasers Med. Sci. 2018;33:1197-1205.

10. Franz A, Behringer M, Nosaka K, et al. Mechanisms underpinning protection against eccentric exercise-induced muscle damage by ischemic preconditioning. Med. Hypotheses. 2017;98:21-27.

11. Salvador AF, De Aguiar RA, Lisbôa FD, Pereira KL, De Cruz RSO, Caputo F. Ischemic preconditioning and exercise performance: A systematic review and meta-analysis. Int. J Sports Physiol. Perform. 2016;11:4-14.

12. Sharma V, Marsh R, Cunniffe B, Cardinale M, Yellon DM, Davidson SM. From Protecting the Heart to Improving Athletic Performance - the Benefits of Local and Remote Ischaemic Preconditioning. Cardiovasc. Drugs Ther. 2015;29:573-588.

13. Incognito A V., Burr JF, Millar PJ. The Effects of Ischemic Preconditioning on Human Exercise Performance. Sports Med. 2016;46:531-44.
14. Beaven CM, Cook CJ, Kilduff L, Drawer S, Gill N. Intermittent lower-limb occlusion enhances recovery after strenuous exercise. Appl. Physiol. Nutr. Metab. 2012;37:1132-1139.

15. Sabino-Carvalho JL, Lopes TR, Obeid-Freitas T, et al. Effect of Ischemic Preconditioning on Endurance Performance Does Not Surpass Placebo. Med. Sci. Sports Exerc. 2017;49:124-132.

16. Franz A, Behringer M, Harmsen JF, et al. Ischemic Preconditioning Blunts Muscle Damage Responses Induced by Eccentric Exercise. Med. Sci. Sports Exerc. 2018;50:109-115.

17. Marocolo M, da Mota GR, Simim MAM, Appell Coriolano H-J. Myths and Facts About the Effects of Ischemic Preconditioning on Performance. Int. J Sports Med. 2016;37:87-96.

18. Bezerra de Morais AT, Cerqueira MS, Moreira Sales R, Rocha T, Galvão de Moura Filho A. Upper limbs total occlusion pressure assessment: Doppler ultrasound reproducibility and determination of predictive variables. Clin. Physiol. and Funct. Imaging. 2017;37:437-441.

19. Loenneke JP, Allen KM, Mouser JG, et al. Blood flow restriction in the upper and lower limbs is predicted by limb circumference and systolic blood pressure. Eur. J Appl. Physiol. 2015;115:397-405.

20. Ferreira TN, Sabino-Carvalho JLC, Lopes TR, et al. Ischemic Preconditioning and Repeated Sprint Swimming: A Placebo and Nocebo Study. Med. Sci. Sports Exerc. 2016;48:1967-75.

21. Damas F, Nosaka K, Libardi C, Chen T, Ugrinowitsch C. Susceptibility to Exercise-Induced Muscle Damage: a Cluster Analysis with a Large Sample. Int. J Sports Med. 2016;37:633-640.

22. Aagaard P, Simonsen EB, Andersen JL, Magnusson P, Dyhre-Poulsen P. Increased rate of force development and neural drive of human skeletal muscle following resistance training. J Appl. Physiol. 2002;93:1318-1326.

23. Peñailillo L, Blazevich $A$, Numazawa $H$, Nosaka K. Rate of force development as a measure of muscle damage. Scand. J Med. Sci. Sports. 2015;25:417-427.

24. Chan A-W, Tetzlaff JM, Altman DG, et al. SPIRIT 2013 Statement: Defining Standard Protocol Items for Clinical Trials. Ann. Intern. Med. 2013;158:200-7.

25. Fuzari HKB, de Andrade AD, Cerqueira MS, et al. Whole body vibration to attenuate reduction of explosive force in chronic kidney disease patients: a randomized controlled trial. J. Exerc. Rehabil. 2018;14:883-890.

26. Chen TC, Chen HL, Lin MJ, Wu CJ, Nosaka K. Potent protective effect conferred by four bouts of low-intensity eccentric exercise. Med. Sci. Sports Exerc. 2010;42:1004-1012.

27. Ferraresi C, De Brito Oliveira T, De Oliveira Zafalon L, et al. Effects of low level laser therapy $(808 \mathrm{~nm}$ ) on physical strength training in humans. Lasers Med. Sci. 2011;26:349-358.

28. Barbosa TC, Machado AC, Braz ID, et al. Remote ischemic preconditioning delays fatigue development during handgrip exercise. Scand. J Med. Sci. Sports. 2015;25:356-64.

29. Seeger JPH, Timmers S, Ploegmakers DJM, Cable NT, Hopman MTE, Thijssen DHJ. Is delayed ischemic preconditioning as effective on running performance during a $5 \mathrm{~km}$ time trial as acute IPC? J Sci. Med. Sport. 2016;20:208-212.

30. Baroni BM, Junior ECPL, Marchi T De, et al. Low level laser therapy before eccentric exercise reduces muscle damage markers in humans. Eur. J Appl. Physiol. 2010;110:789-796.

31. Foster C, Florhaug JA, Franklin J, et al. A New Approach to Monitoring Exercise Training. J Strength Cond. Res. 2001;15:109-15.

32. Tseng K-W, Tseng W-C, Lin M-J, Chen H-L, Nosaka K, Chen TC. Protective effect by maximal isometric contractions against maximal eccentric exercise-induced muscle damage of the knee extensors. Res. Sports Med. 2016;24:228-241. 